\title{
Und wenn das Anordnungsmodell eine Chance wäre?
}

\section{Gebhard Hüsler}

PD. Dr. phil. lic.iur. , Fachpsychologe für Psychotherapie \& Gesundheitspsychologie FSP, ZRG Zentrum für Rehabilitation und Gesundheitspsychologie

Das Anordnungsmodell wurde in den letzten Monaten in der Schweizerischen Ärztezeitung wenig freundlich kommentiert. Betont wurden fast ausnahmslos die möglichen negativen Folgen. Einige Gründe sprechen jedoch für die bessere Verankerung und Zugänglichkeit von Psychotherapie in der Grundversicherung. Es gibt kaum ein Krankheitsgeschehen, das nicht auch unter dem Aspekt psychischer Beeinträchtigung gesehen werden muss.

Die Kommentare bzw. Argumente gegen das Anordnungsmodell lassen sich in zwei Gruppen einteilen: Einerseits wird von den unvorhersehbaren Kosten gewarnt, anderseits wird die fehlende Kompetenz psychologischer Psychotherapeut/-innen ins Feld geführt. Aufgrund der Mengenausweitung und der bevorzugten Behandlung von «Wellnessbehandlung - und Life Coaching Patienten" soll es zu einer Kostenexplosion und zu einer schlechteren Versorgung der «tatsächlichen Patienten» kommen. Erstaunlich ist auch die Heftigkeit der Kommentare angesichts der Tatsache, dass das Delegationsmodell von vorneherein als Provisorium gedacht war.

Das Delegationsmodell hat eine Ausnahmestellung im Rahmen der OKP. Es besteht seit 1981 aufgrund eines Bundesgerichtsentscheids (BGE 107 V 46) und wurde von diesem explizit als Übergangsregelung bezeichnet bis die Berufe der psychologischen Psychotherapeuten und -therapeutinnen gesetzlich geregelt ist (S 4/22, Änderung der Verordnung vom 27. Juni 1995).

Das PsyG ist seit 2013 in Kraft. Deshalb ist dieses Provisorium durch ein anderes Modell zu ersetzen. Zur Kostenausweitung stellt die Änderung der Verordnung bzgl. Neuregelung der psychologischen Psychotherapie fest (Juni, 2019):

Mehrkosten für die OKP entstehen im Wesentlichen durch die Verlagerung von heute privat oder über Zusatzversicherungen bezahlten Leistungen.... mit dem Wechsel zum umfassenden Anordnungsmodell wird das PsyG neu als Voraussetzung der Zulassung zur Leistungsabrechnung festgelegt, wodurch sich der Kreis der Leistungserbringer und Leistungserbringerinnen gegenüber heute verkleinert... es wird von einem Rückgang von 10\% ausgegangen.. ..langfristig wird erwartet, dass sich der Effekt des Wegfalls der nicht PsyG-konform weitergebildeter Psychotherapeuten und -therapeutinnen durch PsyG-konform weitergebildeten Personen kompensiert und eine Mengenzunahme gegenüber heute im Sinne einer Verbesserung der Versorgungssituation im Rahmen von 10\% liegen kann, was zu jährlichen Mehrkosten von CHF 167 Mio führen würde (14/22, Änderung der Verordnung vom 27. Juni 1995).

Dabei handelt es sich um eine Investition in die $\mathrm{Zu}-$ kunft!

Die bessere Verankerung und Zugänglichkeit von Psychotherapie in der Grundversicherung soll dazu beitragen, die Versorgung zu optimieren und Kosten aufgrund der Nichtbehandlung zu senken. Im Detail würde der Einschluss psychologischer Dienstleistungen in die obligatorische Grundversicherung über 40 Jahre zu einer signifikant geringeren Lebenszeitprävalenz psychiatrischer Hospitalisierungen ..., weniger Suizidversuchen und weniger Suizidtoten führen (S. 13,14/22 Änderung der Verordnung vom 27. Juni 1995)

Eine Verankerung in der Grundversorgung ist dringend geboten, da bis anhin weite Bevölkerungsgruppen aufgrund mangelnder finanzieller Mittel keine Psychotherapie in Anspruch nehmen konnte und auf die «billigere Lösung» einer Psychomedikation verwiesen wurde. Hier wäre allenfalls noch die beeindruckende Mengenausweitung der Psychopharmaka in Europa zu erwähnen. Ohne die Psychomedikation verteufeln zu wollen, scheint nach den letzten Publikationen [1] zumindest bei den Antidepressiva eine Wirksamkeit in Frage gestellt. Nicht bestritten ist, dass bei allen psychischen Störungen, selbst wenn eine Psychomedikation erfolgt, eine begleitende Psychotherapie empfohlen wird und Publikationen zeigen, dass die Kombination jedenfalls bessere Resultate erzielt. Die gegenwärtige Annahme, dass Antidepressiva allenfalls bei schweren Depressionen wirken ist seit 2008 bekannt [2]. Diese Erkenntnis verlangt gerade zu, dass 


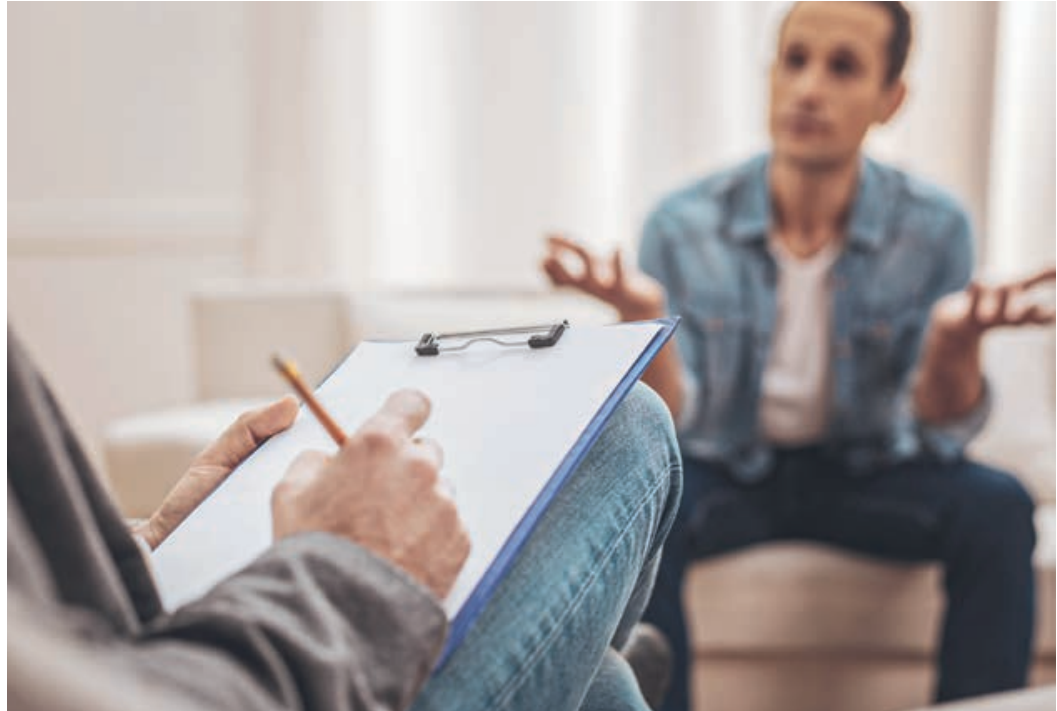

Psychotherapie sollte niederschwelliger angeboten werden.

(Symbolbild, (C) Viacheslav lacobchuk | Dreamstime.com)

Psychotherapie niederschwelliger angeboten werden muss. Betrachtet man das Krankheitsgeschehen von einem sozioökonomischen Standpunkt aus, dann hat das untere Drittel auf der sozioökonomischen Leiter ein dreifach höheres Risiko eine psychische Krankheit zu erleiden, als das oberste Drittel [3]. Bedingt durch die Kumulation der Probleme, wie Arbeitslosigkeit, Scheidung, Krankheit kommt es zu Schwierigkeiten in anderen Bereichen wie fehlende finanzielle Mittel, die dann zu einem psychischen Druck führen, der dann oft in eine psychische Störung führt. Jeder Case Manager einer beliebigen Krankenkasse kann ein Lied davon singen. Deshalb ist auch das ins Feld führen des «biospsychosozialen Modells» mit der Betonung auf «bio» fehl am Platz. Die sozialen Komponenten psychischer Erkrankungen werden ganz grundsätzlich unterschätzt. Die Diskussion um die Entstehung psychischer Erkrankungen flammt in diesem Zusammenhang immer wieder auf. Die Annahme, dass es sich um "Hirnerkrankungen" handelt, wird vorab

\section{Die sozialen Komponenten psychischer Erkrankungen werden ganz grundsätzlich unterschätzt.}

von den «Biologisten» favorisiert. Im Zeitalter des Gehirns herrscht der Glaube vor, wenn wir verstehen, wie das neuronale Netz arbeitet und Reize verarbeitet, die einzelnen Funktionen des Gehirns beschreiben können und die Neuromodulatoren und Neurotransmitter verstanden haben, dann lassen sich «Entgleisungen» also das nicht "normierte Hirn", schlussendlich als Stoffwechsel Problem behandeln. Dann müssen nur noch die dementsprechenden Substanzen, sei es als
Nahrungssubstrat, sei es als Psychopharmakon bereit gestellt werden und alles kommt wieder ins Lot. Hier fallen Gehirnfunktionen und Psyche zusammen. Mein Hirn sagt mir, wer ich bin. Wohltuend in diesem $\mathrm{Zu}^{-}$ sammenhang war das Interview mit Prof. D. Zullino im Swiss Archives of Neurology, Psychiatry and Psychotherapie: Brauchen wir eine neue Psychiatrie-Re-Evoluation? [4] Herr Zullino kommt in diesem Interview zum Schluss, dass es "einen relativen Stillstand» in der aktuellen Psychiatrie gäbe. Zwar sei das neurobiologische Wissen exponentiell gewachsen, aber zu einer Umwälzung in der Psychiatrie habe es nicht geführt. Er plädiert für eine Öffnung der Psychiatrie und der Zusammenarbeit mit anderen Berufsgruppen. Da wäre auch noch ein Zitat von Dürrenmatt ins Feld zu führen. Was alle angeht, können nur alle lösen. Die Zentrierung auf die «Medikalisierung» sozialer und psychischer Probleme wird ja des Öfteren beklagt. Die Technikerkrankenkasse aus der BRD schrieb in einer Publikation "Sind wir heute anders krank?» Ja wir sind anders krank [5]. Es gibt kaum ein Krankheitsgeschehen das nicht auch unter dem Aspekt psychischer Beeinträchtigungen gesehen werden muss. Vielleicht lässt sich das bio-psycho soziale Modell einmal umformulieren in ein sozio psycho biologisches Modell. Das Ausspielen von tatsächlich psychisch Kranken und

Es gibt kaum ein Krankheitsgeschehen das nicht auch unter dem Aspekt psychischer Beeinträchtigungen gesehen werden muss.

vermeintlich psychisch Kranken ist auf diesem Hintergrund kaum nachvollziehbar.

Die Chancen des Anordnungsmodells erlauben eine Zusammenarbeit «auf Augenhöhe» mit anderen Disziplinen. Die in Frage gestellte fachliche Kompetenz psychologischer Psychotherapeuten, die verschiedentlich in den Kommentaren aufgeworfen wurde, nimmt unhinterfragt an, dass nur eine medizinische Ausbildung befähigt, die gesamte Situation eines Patienten einzuschätzen. Das ist ein Irrtum und geht bereits an der gegenwärtigen Realität vorbei. In einer arbeitsteiligen Welt ist es schon lange eine Selbstverständlichkeit, dass bspsw. der Hausarzt Fachkräfte aus der Medizin oder anderen Disziplinen zu Rate zieht, wenn er an seine Wissens- oder Erfahrungsgrenzen stösst. Dass gerade der Hausarzt als die niederschwelligste Instanz im Krankheitsgeschehen beim Anordnungsmodell nicht als die zuweisende Instanz tätig sein soll, ist störend und sollte nachgebessert werden. Das Hausarztmodell wird nur mit dieser zusätzlichen Kompetenz seine Wirkung voll entfalten können. Dass den Hausärzten ohne Zusatzausbildung diese Kompetenz verwehrt wird, ist 
Gebhard Hüsler

PD. Dr. phil. lic. iur.

Fachpsychologe für Psycho therapie \& Gesundheitspsychologie FSP

ZRG Zentrum für Rehabilitation und Gesundheitspsy-

chologie, Place de la Gare 5, CH-1700 Fribourg

Telefon +41 (0)79 6694559

E-Mail ghuesler[at]zrg.ch vermutlich der Befürchtung zuzuschreiben, dass eine unkoordinierte Mengenausweitung stattfinden würde. Ob diese Befürchtung zutrifft, kann durch eine weitere Vorgabe des Anordnungsmodells geprüft werden. Das Modell sieht vor, dass jede ärztliche und psychologische Psychotherapie mit einer Einstiegs-Verlaufs-und Erfolgsdiagnostik begleitet wird. Damit lässt sich endlich, was für die Schweiz fehlt, eine Angabe über Ausmass und Schweregrad psychischer Störungen machen. Längerfristig würde sich hier auch prüfen lassen, ob Psychotherapie nicht nur psychisches Leid mildert, sondern sich generell förderlich auf die Gesellschaft auswirkt, indem schwierige berufliche und soziale Situ- ationen schneller gelöst oder dann sogar verhindert werden können und die Lebensqualität verbessert.

\section{Literatur}

1 Munkholm K, et al. BMJ Open 2019;9:e024886. doi:10.1136/ bmjopen-2018-024886.

2 Kirsch I et al. PLoS Med 2008; 5 (2): e45

3 Hüsler, G. Chronifizierende Krankheiten - Psychosoziale Implikationen, Psychodynamische Psychotherapie (im Druck), 2019, PDP.

4 Zullino Daniele, Studer Karl. Brauchen wir eine neue PsychiatrieRe-Evoluation? Swiss Arch Neurol Psychiatr Psychother. 2019;170:w03038.

5 Hüsler, G. Sind wir heute anders krank? Psychodynamische Psychotherapie. 2014, PDP, 13:139-151.

\section{Das Wichtigste in Kürze}

- Die Verankerung der Psychotherapie in der Grundversorgung ist dringend geboten; Bei allen psychischen Erkrankungen zeigt eine die Medikation begleitende Psychotherapie bessere Resultate als Medikation alleine.

- Vom sozioökonomischen Standpunkt aus hat das untere Drittel ein dreifach höheres Risiko, eine psychische Krankheit zu erleiden. Diese Bevölkerungsgruppe kann aufgrund mangelnder finanzieller Mittel oft keine Psychotherapie in Anspruch nehmen. Soziale Komponenten psychischer Erkrankungen werden ganz grundsätzlich unterschätzt.

- Das Anordnungsmodell erlaubt eine Zusammenarbeit "auf Augenhöhe» mit anderen Disziplinen. Es gibt kaum ein
Krankheitsgeschehen, das nicht auch unter dem Aspekt psychischer Beeinträchtigung gesehen werden muss.

- Krankenkassenverbände argumentieren gegen das Anordnungsmodell mit einer Mengen- bzw. Kostenexplosion. Das Anordnungsmodell sieht sowohl für ärztliche als auch nicht ärztliche Psychotherapeut/innen eine Eingangs- und Verlaufsdiagnostik für die Patient/innen vor. Damit kommen Anzahl und Häufigkeit psychischer Erkrankungen als auch Kosten und Nutzen aus der Grauzone der Spekulation heraus.

- Als niederschwelligste Instanz im Krankheitsgeschehen sollte der Hausarzt der Zuweiser im Anordnungsmodell sein.

\section{L'essentiel en bref}

- L'ancrage de la psychothérapie dans les soins de base est urgent. La psychothérapie en complément des médicaments donne de meilleurs résultats que les médicaments seuls contre toutes les affections psychiques.

- D'un point de vue socio-économique, le tiers le moins aisé de la population a un risque trois fois plus élevé de souffrir d'une affection psychique. Cette catégorie n'a souvent pas les moyens de recourir à une psychothérapie. Les composantes sociales des affections psychiques sont fondamentalement sous-estimées.

- Les opportunités qu'offre le modèle de la prescription permettent une collaboration "d'égal à égal» avec d'autres disciplines. Rares sont les maladies qui ne doivent pas également être envisagées sous l'angle de l'atteinte psychique.
- Les associations d'assureurs-maladie s'opposent au modèle de la prescription en arguant d'une explosion de la quantité et des coûts. Ce modèle prévoit un diagnostic initial et d'évolution pour les patient-e-s, tant dans le cas des psychothérapeutes médecin que non-médecins. Le nombre et la fréquence des affections psychiques, de même que les coûts et les bénéfices, sortent ainsi de la zone grise des spéculations.

- Le médecin de famille étant l'instance la plus accessible dans le cadre de la maladie, il devrait être le référent dans le modèle de la prescription. Le modèle du médecin de famille pourrait ainsi contribuer à une meilleure prise en charge, donc à une réduction des coûts à long terme. 